\title{
Development of a combined OCT-Raman probe for the prospective in vivo clinical melanoma skin cancer screening
}

M. Mazurenka, L. Behrendt, M. Meinhardt-Wollweber, U. Morgner, and B. Roth

Citation: Review of Scientific Instruments 88, 105103 (2017); doi: 10.1063/1.5004999

View online: https://doi.org/10.1063/1.5004999

View Table of Contents: http://aip.scitation.org/toc/rsi/88/10

Published by the American Institute of Physics

\section{Articles you may be interested in}

Note: Double-hole cantilevers for harmonic atomic force microscopy

Review of Scientific Instruments 88, 106101 (2017); 10.1063/1.4991073

Design and test of a simple fast electromagnetic inductive gas valve for planar pulsed inductive plasma thruster

Review of Scientific Instruments 88, 105101 (2017); 10.1063/1.4995234

A large-diameter hollow-shaft cryogenic motor based on a superconducting magnetic bearing for millimeterwave polarimetry

Review of Scientific Instruments 88, 105102 (2017); 10.1063/1.4990884

A flow cell for the study of gas-solid reactions via in situ powder X-ray diffraction

Review of Scientific Instruments 88, 105104 (2017); 10.1063/1.4996940

A temperature compensated dielectric test cell for accurately measuring the complex permittivity of liquids

Review of Scientific Instruments 88, 105105 (2017); 10.1063/1.5005857

Note: A modified optics based technique for suppressing spurious signals in photoreflectance spectra Review of Scientific Instruments 88, 106103 (2017); 10.1063/1.4993263

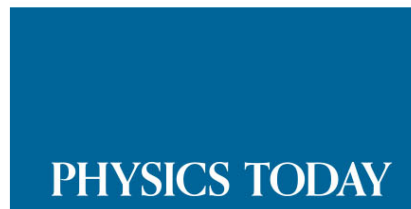

MANAGER'S GUIDE

WHITEPAPERS
Accelerate R\&D with

Multiphysics Simulation

\section{READ NOW}

PRESENTED BY

$\checkmark \subset \bigcirc M S O L$ 


\title{
Development of a combined OCT-Raman probe for the prospective in vivo clinical melanoma skin cancer screening
}

\author{
M. Mazurenka, ${ }^{1, a)}$ L. Behrendt, ${ }^{1}$ M. Meinhardt-Wollweber, ${ }^{1}$ U. Morgner, ${ }^{2}$ and B. Roth ${ }^{1}$ \\ ${ }^{1}$ Hannover Centre for Optical Technologies (HOT), Leibniz Universität Hannover, Nienburger Str. 17, \\ 30167 Hanover, Germany \\ ${ }^{2}$ Institut für Quantenoptik, Universität Hannover, Welfengarten 1, 30167 Hanover, Germany
}

(Received 28 April 2017; accepted 15 September 2017; published online 4 October 2017)

\begin{abstract}
A combined optical coherence tomography (OCT)-Raman probe was designed and built into a spectral domain OCT head, and its performance was evaluated and compared to the most common Raman probe setups, based on a fiber bundle and confocal free space optics. Due to the use of the full field of view of an OCT scanning lens, the combined probe has a superior performance within maximum permissible exposure limits, compared to the other two probes. Skin Raman spectra, recorded in vivo, further prove the feasibility of the OCT-Raman probe for the future in vivo clinical applications in skin cancer screening. Published by AIP Publishing. https://doi.org/10.1063/1.5004999
\end{abstract}

\section{INTRODUCTION}

The development of non-invasive methods for melanoma skin cancer screening is of great interest for both scientific and medical communities, as early diagnosis together with complete surgical excision of the cancerous tumor is essential for improving of the patients' prognosis. Currently melanoma skin cancer screening consists of an initial visual inspection, usually resulting in a large number of false positives, and past-excisional histopathological diagnosis, which is invasive and time consuming. If cancer (melanoma) is confirmed, an additional surgery is often required to reduce the risk of recurrence. To minimize the rate of recurrence, establishing adequate surgical margins (currently $1 \mathrm{~cm}$ for the melanoma Breslow's depth of $\leq 1-2 \mathrm{~mm}$ and $2 \mathrm{~cm}$ for the melanoma Breslow's depth of 2.01-4.0 mm) for the first surgery is important. Thus, the development of methods for the precise presurgical determination of 3D tumor dimensions and, hence, less aggressive treatment of lesions is highly relevant in this field. As the number of melanoma skin cancer incidence worldwide constantly increases, there is an urgent need for a fast, noninvasive, and objective technique for preoperative melanoma skin cancer diagnosis and margin assessment for the further excision.

A number of non-invasive optical diagnostic modalities have been applied for skin cancer detection so far: fluorescence spectrometry, Raman spectroscopy, confocal microscopy, optical coherence tomography (OCT), and reflectance spectrometry, thoroughly reviewed in Ref. 1 . None of these techniques excels in both diagnostics and margin assessment. However, several complementary modalities could be used simultaneously in the attempt to achieve the fast in vivo diagnosis and margin assessment.

Out of all optical non-invasive modalities, OCT is the most applicable for margin detection and also provides morphological information, while Raman spectroscopy is the most

\footnotetext{
a)Author to whom correspondence should be addressed: mikhail.mazurenka@ hot.uni-hannover.de
}

promising technique not only for distinguishing cancerous tumors from the benign ones but also for the identification of the skin cancer types. Co-localized measurements with a combination of OCT and Raman modalities have been applied for cancer diagnostics $^{2-6}$ and have shown a substantial increase in sensitivity and selectivity compared to both these modalities used separately. ${ }^{6}$

OCT is a well-developed technique, and commercially available OCT devices can easily be used for in vivo measurements in a clinical environment. Raman spectroscopy, on the other hand, is at the stage of development as an in vivo technique for skin cancer diagnostics. ${ }^{7}$ For fast in vivo clinical Raman screening, a relatively small hand-held Raman probe is required in order to reach skin areas on all parts of the human body, and an optical fiber bundle with a light delivery fiber and several detection fibers is an obvious choice for such a Raman probe. However, both ex vivo and in vivo Raman measurements have shown that the detection of the Raman signal from the biological tissue is not a trivial task. The tissue Raman spectrum is usually masked by a fluorescence signal from the tissue itself. In addition, light delivered to the tissue from the light source may produce a substantial luminescence signal, due to the crystalline impurities within the fiber material, and also a Raman signal while propagating in the source fiber. These signals are then scattered by the tissue surface into the detection fibers. Moreover, the Rayleigh scattered light of the incident wavelength also produces luminescence and Raman signals within the detection fibers of the bundle. To eliminate the spectral contamination from luminescence and Raman signals, generated within the fibers of the Raman fiber bundle probe, an in-the-tip laser line filter may be used at the exit end of the source fiber, along with in-the-tip notch or long-pass filters on the detection fibers to prevent the Rayleigh scattered light from entering these fibers. ${ }^{8}$ A similar effect can be achieved with the use of hollow fibers. ${ }^{9}$ Another approach for minimizing the influence of the in-fiber generated Raman signals is to perform measurements at high wave numbers $\left(>2000 \mathrm{~cm}^{-1}\right.$ where no fiber Raman peaks are present ${ }^{10}$ ). To lessen the tissue luminescence signal, shifted subtracted Raman spectroscopy 
(where two, slightly shifted, Raman spectra are recorded and then subtracted one from another, thus leaving a Raman spectrum without luminescence background ${ }^{11}$ ) and the wavelength modulation can be employed.

Another drawback of using fiber bundles as Raman probes is a poor overlap of the illuminated sample volume with the sample volume from which the signal is collected due to the low numerical aperture (NA) of the optical fibers. This can be improved by beveling collection fibers to increase the overlap of the collection cones with the source cone ${ }^{8}$ or by placing the fiber bundle $1-3 \mathrm{~mm}$ above the sample. ${ }^{12}$ A ball lens attached to the fiber tip to increase the collection efficiency ${ }^{9,13}$ has also been used, as well as a free space optic probe with line and low-pass filters built in Refs. 7, 14, and 15. The challenges in the developing of the in vivo Raman probes are in depth reviewed by Stevens et al. ${ }^{16}$ and Pence and Mahadevan-Jansen. ${ }^{17}$ Despite all these approaches to minimize contamination and to increase the signal-to-noise ratio (SNR) of the skin Raman spectra, the Raman signal is often still too low to be detected reliably, unless high power densities of the incident light, sometimes exceeding Maximum Permissible Exposure (MPE) values by several orders of magnitude, are used, as summarized in Ref. 7. This restricts the applicability of Raman spectroscopy from routine clinical in vivo measurements on human skin. We have found that the group of Zeng and Lui is particularly concerned with the issue of MPE values and took them into consideration for designing, building, and clinical use of their in vivo free-space confocal Raman probe, see Ref. 14, for instance.

In this letter, we show that distributing the laser power over the full field of view of a scanning OCT lens allows recording of a Raman spectrum from human skin in vivo at skin irradiance below MPE values with a good SNR. We also assess the performance of our combined OCT-Raman probe and compare it to the performance of other Raman probes, in particular, a fiber bundle probe and a confocal probe. The components required for Raman spectroscopy were incorporated in such a way in the OCT system that the OCT performance is not affected.

\section{COMBINED OCT-RAMAN SETUP DESIGN}

The schematic for the OCT-Raman setup is shown in Fig. 1(a). The spectral domain OCT device (Telesto II, Thorlabs) consists of a base unit and a scientific, cage mounted, OCT head equipped with an LSM03 scan lens, which was modified for the detection of the Raman signal. The OCT signal collection part of the head was not changed so that the OCT imaging capabilities of the head were not compromised. We only removed the probe alignment CCD camera of OCT and used this port to guide the collected Raman light to the spectrometer, Fig. 1(b). The OCT and Raman signals were collected one after another rather than simultaneously in this configuration. Therefore, all available OCT scan modes are the same as in the original instrument and can also be applied to other types of OCT devices.

Figure 1(c) shows a raw OCT image of a melanocytic lesion on skin, recorded with our setup at $10 \mathrm{kHz}$ A-scan line rate with $20 \mathrm{~A}$-scan and no B-scan averaging. The OCT $\mathrm{SNR}$, calculated as $\mathrm{SNR}=20 \times \log _{10}\left(\mathrm{E}_{\mathrm{M}} / \mathrm{E}_{\mathrm{N}}\right)$, is equal to $76 \mathrm{~dB}\left(\mathrm{E}_{\mathrm{M}}\right.$-maximum of an A-scan signal and $\mathrm{E}_{\mathrm{N}}$ - standard deviation of signal in the A-scan). The suitability of the OCT head for the melanocytic lesion depth determination was investigated earlier ${ }^{18}$ in comparison with high-frequency ultrasound.

An adaptor for in vivo Raman measurements [Fig. 1(b)] was designed and printed on a 3D printer (MakerBot Replicator $2 \times$ with strong black premium ABS filament, a)

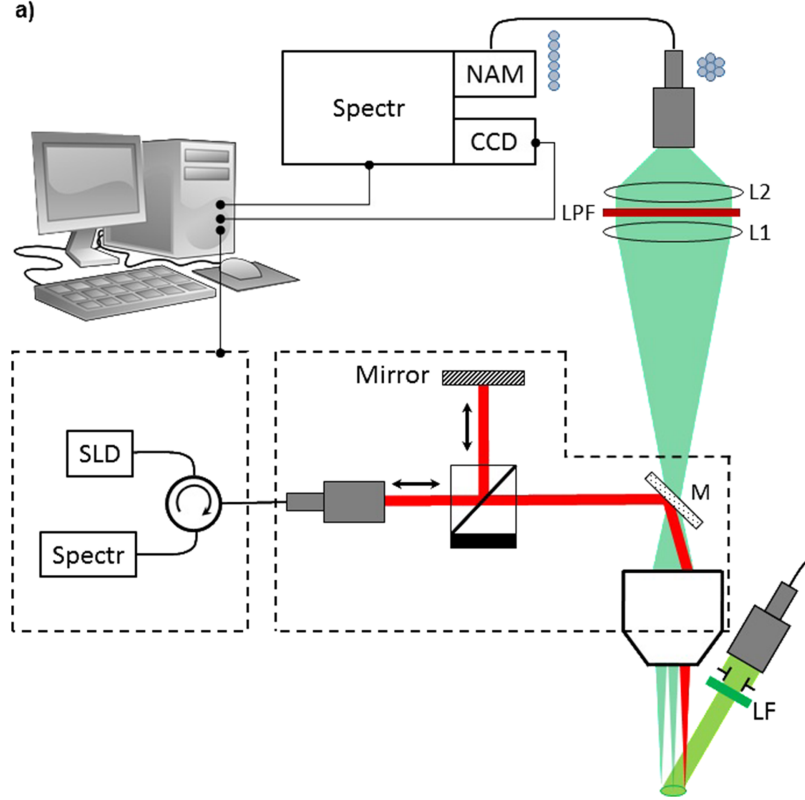

b)

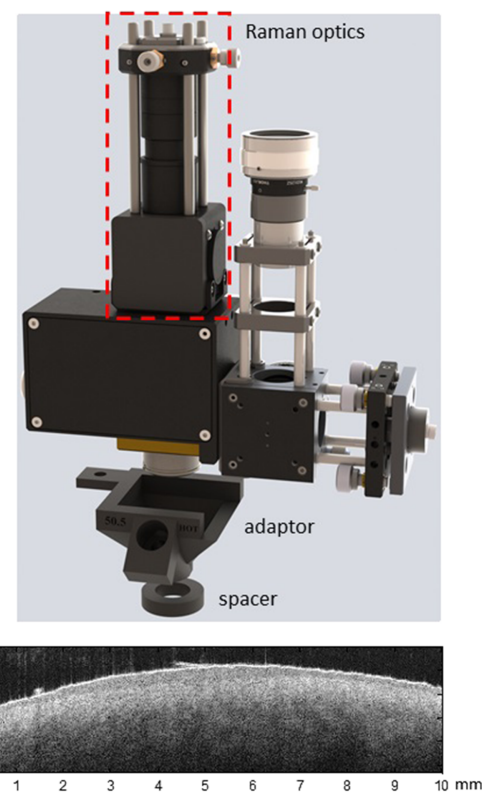

FIG. 1. Experimental setups of the combined OCT-Raman setup (a); 3D CAD design of a scientific, cage mounted OCT with attached Raman optics, an adaptor, and a spacer (b); a raw OCT image of a skin lesion, recorded at $10 \mathrm{kHz}$ A-scan line rate without postprocessing (c). LF-laser line filter, LPF-long-pass filter, $\mathrm{L}$-lens, $\mathrm{M}$-mirror, $\mathrm{DM}$ - dichroic mirror, and $\mathrm{M}$-scanning mirrors. 
3Dmensionals). The adaptor serves two main purposes: it holds an illumination arm of the Raman setup and protects the sample area from the room light present at clinical measurements. A number of rubber spacers were also printed (NinjaFlex filament, 3Dmensionals). The rubber was chosen due to its flexibility, which allows better contact with the skin on the parts of body with different topography, thus, preventing the room light from leaking into the detection arm of the Raman setup, it also provides better comfort for the patients during the measurements. The height of the adaptor was set to have the imaged surface around $100 \mu \mathrm{m}$ further than the working distance of the imaging lens. The incident laser beam of the Raman setup was aligned to illuminate the center of the imaged surface at the distance set by the adaptor height. During the in vivo measurements, a spacer [Fig. 1(b)] is attached to the adaptor to place the top of the lesion being measured to the distance the laser beam is aligned to. The thickness of the spacer is chosen by observing the OCT scans, thus insuring the collection of the Raman signal from the top of the lesion. The spacer thickness ranges from $0 \mathrm{~mm}$ to $7.6 \mathrm{~mm}$ with steps of $0.4 \mathrm{~mm}$.

For the Raman measurements, light from a light source (Ultra 50, Quantel; $532 \mathrm{~nm}, 7 \mathrm{~ns}$ pulse duration at $20 \mathrm{~Hz}$ repetition rate) is delivered by a fiber and collimated to the tissue to illuminate a maximal area of the elliptic shape with axis dimensions of $9 \mathrm{~mm}$ and $7 \mathrm{~mm}$ to fill most of the full field of view of the OCT scanning lens. The pulsed $532 \mathrm{~nm}$ laser was chosen due to the planned further combination of the OCT-Raman head with an optoacoustic detector working with $532 \mathrm{~nm}$ pulsed excitation light to measure tumor depths larger than $1 \mathrm{~mm}$, which is the limiting depth for the OCT in skin. Thus, in order to combine the three modalities, OCT, Raman, and optoacoustics in future, minimizing the number of laser sources and optical components is required and using standard optics, i.e., the OCT components designed to work at wavelengths around $1325 \mathrm{~nm}$, and compact laser sources are important aspects. The $532 \mathrm{~nm}$ wavelength is also of interest of dermatologists, as $\beta$-carotene Raman resonance lines start to appear in the skin spectrum at this wavelength, as shown later in the recorded Raman spectra, potentially providing additional information for the tumor composition analysis. Compared to other wavelength ranges used for Raman spectroscopy of skin, in particular in the near-infrared region, at $532 \mathrm{~nm}$ not only the luminescence increases (which can be subtracted in the data processing step) but also the Raman signal itself which is advantageous to improve the signal-tonoise-ratio of the spectra. Moreover, the skin absorption in the near-infrared is smaller than at $532 \mathrm{~nm}$, allowing the light to penetrate deeper into the tissue ( $\mathrm{cm}$ range). This might mean that a portion of the detected Raman signal may originate from the tissue below the tumor, especially in the case of thin tumors $(<1 \mathrm{~mm})$, and might necessitate further studies.

An iris is used to reduce the illuminated area when the lesion is smaller than $9 \times 7 \mathrm{~mm}$. The position, relative to a lesion, and size of the illuminated area are controlled via a camera. We use the same camera that was built into the OCT head, as it does not require additional software. A $532 \mathrm{~nm}$ laser line filter (Thorlabs) was used to clean up the excitation light from luminescence, and Raman signals were generated within the source fiber. Light scattered and emitted by the tissue is collected by the OCT scanning lens, and its exit pupil, situated at the position of the scanning mirrors [schematically shown in Fig. 1(a) as mirror M], is imaged onto a detection fiber bundle by two lenses with foci of $125 \mathrm{~mm}$ and $25.4 \mathrm{~mm}$, respectively. The lenses are chosen to maximize the light coupling into the fiber bundle, as was optimized with Zemax simulations for commercially available lenses. A long-pass filter (532 nm RazorEdge ultrasteep LP Edge filter, Semrock, Inc.) was placed between lenses to prevent Rayleigh scattered light from entering the detection fiber bundle. The scanning mirrors of the OCT head are coated to have maximum reflectivity around $1310 \mathrm{~nm}$, the working wavelength of the OCT, while at $532 \mathrm{~nm}$ mirror transmission is about $95 \%$, as measured by Thorlabs. The detection fiber bundle is a custom made (Ceramoptec) fiber bundle, consisting of 18 fibers (each Ø $200 \mu \mathrm{m}$ and NA $=0.22$ ), arranged linearly on one end and into a circle (round, $\varnothing 1.2 \mathrm{~mm}$ ) on the other end. The linear end of the fiber bundle was designed to fit a NA matcher attached to a spectrometer (Shamrock SR-500i, Andor).

\section{OCT-RAMAN PROBE PERFORMANCE ASSESSMENT}

The performance of the designed OCT-Raman probe was compared to the most commonly used probes for in vivo Raman measurements, based on a fiber bundle and a confocal setup, both theoretically and experimentally. As the fiber based probe, we used the fiber bundle described above, and to mimic the confocal probe, we used a commercially available high-NA lens, $\varnothing 25.4 \mathrm{~mm}$ and $\mathrm{f}=25.4 \mathrm{~mm}(\mathrm{NA}=0.45)$. All three probes under consideration, each of them standing exemplarily for a configuration commonly used to date in this field, schematically shown in Figs. 2(2-4), were examined in terms of light collection efficiency, which is the simplest first step for system performance assessment. Also, the responsivity of the different probes, i.e., the ratio of the detected photons to the photons emitted by the tissue, is determined and compared.

For this purpose, we assume a Lambertian light source and an ideal optical system, aberration free, with no internal apertures, placed in the air, so that the refractive index of the surrounding medium is equal to 1 . Radiant power (or light flux) $\Phi$ transferred by such an optical system is linearly proportional to the radiance of the light source, $L_{o}$, the area of the light source seen by the optical system, $A_{o}$, and a solid angle this area is visible from, $\Omega_{o}$. This can be easily derived from the paraxial optical theory and is illustrated in Fig. 2(1).

The formula for the radiant power $\Phi$, also displayed in Fig. 2, can be directly applied to the confocal probe design, as shown in Fig. 2(2). However, in the case of the OCT probe, determination of the NA for the scanning lens is not possible. Instead, for a given effective focal length, $z_{o}=36 \mathrm{~mm}$ and exit pupil size, $d=4 \mathrm{~mm}$, and assuming only small angles of incidence (which is realistic), we can calculate the solid angle $\Omega_{o}$ from geometrical consideration as illustrated in Fig. 2(3), assuming the integral detection of the light from the full field of view of the OCT scanning lens. For the fiber bundle probe, attached directly to the light source, the light flux $\Phi$ can be 
1

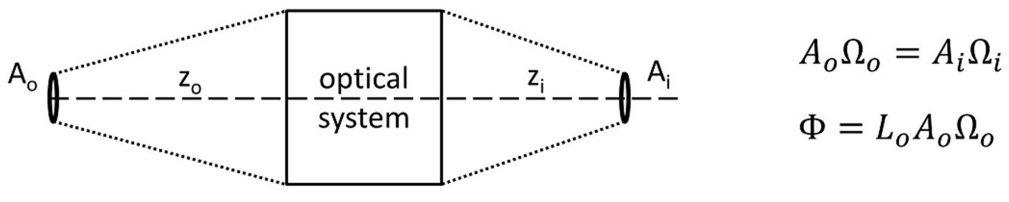

d

2
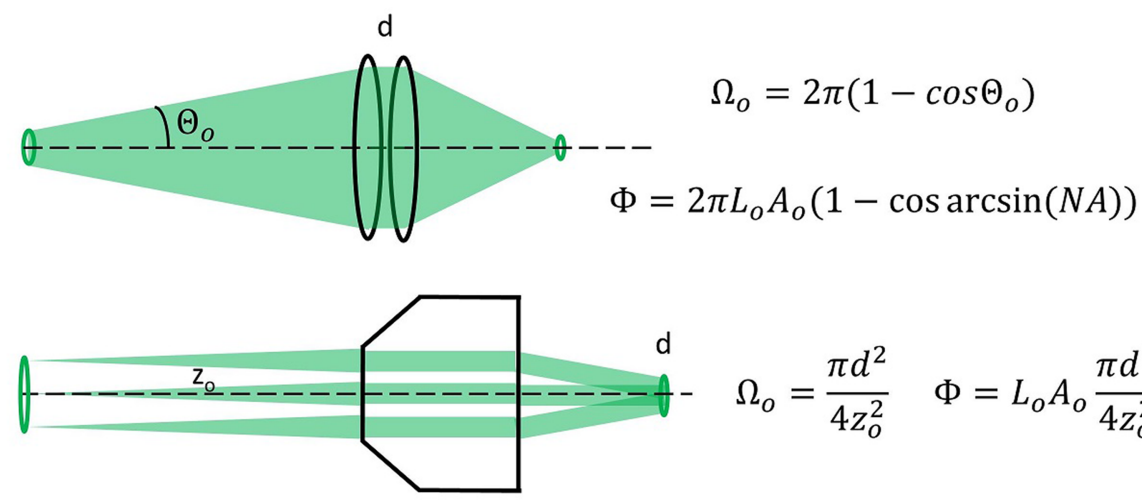

$\Omega_{o}=\frac{\pi d^{2}}{4 z_{o}^{2}} \quad \Phi=L_{o} A_{o} \frac{\pi d^{2}}{4 z_{o}^{2}}$

FIG. 2. A schematic for the Raman probe designs, their photon collection efficiency calculations, and responsivity measurements. (1) An ideal optical system, (2) a confocal probe, (3) an OCT probe, (4) a fiber bundle probe, and (5) a phantom for the experimental Raman probe performance assessment. $A_{o}, A_{i}$ and $z_{o}, z_{i}$ are areas and distances of the object and image, respectively, and $\mathrm{d}$ is the diameter of aperture of the optical system.

4

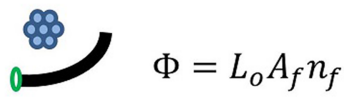

5

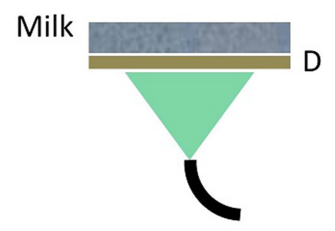

calculated simply by multiplying the source radiance by the area of all detection fibers in the fiber bundle, see Fig. 2(4), where $A_{f}$ is the area of a single fiber and $n_{f}$ is the number of fibers in the fiber bundle.

The detectable light flux, $\Phi$, was calculated for all three probes [Figs. 2(2-4)] and summarized in Table I, together with the optical parameters, relevant for each probe. The $A_{o}$ parameter for the confocal probe depends on the diameter of the detector $A_{i}$, and in our setup, it was measured to be $3.14 \mathrm{~mm}^{2}$. The values for $\Phi$ shown in Table I are normalized on the radiance $L_{o}$. A rough estimation shows that the OCT-Raman probe lens should perform considerably better than our fiber bundle due to much larger photon collection area, thus, indicating the feasibility of using the OCT lens for the in vivo Raman probe design.

In the real setup, there are several sources of optical losses in all three types of Raman probes. The combined OCT-Raman probe would have the largest losses among all three probes due to the limited transmission of the OCT scanning mirrors, reflections from the surfaces within the multicomponent OCT lens, not optimized for the green part of the spectrum, and not optimal Raman signal coupling efficiency, limited by the optical setup of the OCT head. To assess the experimental performance of the OCT-Raman probe, the responsivity measurements were performed for all three setups.

For the Raman probe performance assessment, responsivity can be defined as a ratio between the detected signal and the amount of light emitted by the tissue. Thus, responsivity measurements require a light source with the well-defined radiance. Alternatively, when relative, rather than absolute, responsivity measurements are sufficient for the device characterization purposes, like in our case, any stable light source can be used and, normalized on $L_{o}$, responsivity will yield all necessary information.

As a light source, a milk phantom was used, schematically shown in Fig. 2(5). Laser light was delivered to a $20^{\circ}$ circle tophat engineered diffuser (Thorlabs) and then passed through a $1 \mathrm{~cm}$ thick cuvette with milk producing a light emitting spot of $\sim 3.5 \mathrm{~cm}$ diameter, assuring that the full field of view for the OCT lens $(\varnothing \sim 1 \mathrm{~cm})$ and full NA of the confocal probe are filled. The milk phantoms are often used to mimic the biological tissue. Similar to the real tissue, milk phantoms

TABLE I. Comparison of photon collection efficiency of three types of Raman probes. $\mathrm{A}_{0}$ - the measured surface area, from which each probe collects photons; $\mathrm{z}_{0}$ - the distance between the surface of the phantom and the probe; $\mathrm{d}$ - the diameter of the aperture of the optical system; $\Phi$ - the calculated detectable flux; relative $\Phi$ - the normalized $\Phi$; relative responsivity - the normalized calculated responsivity of each optical system; MPE pulse energy-the MPE valued calculated for skin exposure to $532 \mathrm{~nm}$ pulsed laser light (pulse length of $7 \mathrm{~ns}, 20 \mathrm{~Hz}$ repetition rate) for $10 \mathrm{~s}$.

\begin{tabular}{lccccccc}
\hline \hline Raman probe & $\mathrm{A}_{0}\left(\mathrm{~mm}^{2}\right)$ & $\mathrm{z}_{0}(\mathrm{~mm})$ & $\mathrm{d}(\mathrm{mm})$ & $\Phi\left(\mathrm{s}^{-1}\right)$ & Relative $\Phi$ & $\begin{array}{c}\text { Relative } \\
\text { responsivity }\end{array}$ & $\begin{array}{c}\text { MPE pulse energy } \\
(\mathrm{mJ})\end{array}$ \\
\hline Fiber bundle & 0.57 & 0 & $0.2 \times 18$ & 0.57 & 1 & 1 & 0.039 \\
Confocal & 3.14 & 25.0 & 24.0 & 2.11 & 3.7 & 1.55 & 0.156 \\
OCT-Raman & 69.40 & 25.0 & 4.0 & 1.40 & 2.5 & 1.54 & 3.6 \\
\hline \hline
\end{tabular}


provide output intensity distribution close to the Lambertian and are, thus, ideally suited for the medical device evaluation. For all measurements, a probe was placed above the center of the phantom at phantom-probe distances, $z_{o}$, used in the lens performance estimations and specified in Table I.

The measurements were done in the transmission mode. For that purpose, the long-pass filter was removed from the OCT-Raman probe to allow the detection of $532 \mathrm{~nm}$ light. For the confocal probe, the coupling into the detection fiber bundle was also optimized with Zemax software, and an appropriate lens was used for the measurements. The fiber bundle was used as is. For all three probes, the same detection fiber bundle, optimized for the best performance with the detection spectrometer, was used.

The use of the same light source, the same detection fiber bundle, and the spectrometer ensured that all differences in the Raman probe performance can be attributed only to the probes themselves.

For the responsivity measurements, the signal was integrated for $10 \mathrm{~s}$ and then, after background subtraction, the area under the laser line, recorded by the spectrometer, was integrated.

For the Raman probe performance comparison, it is more convenient to calculate their responsivity relative to the fiber bundle probe, as the one with no additional optics. The relative responsivity values are presented in Table I. To see the difference between estimated and measured performances of the Raman probes, the calculated light flux, $\Phi$, was also presented as relative to the fiber bundle value, see Table I. From Table I, it is evident that the relative experimental performance of the confocal and OCT-Raman probes is substantially lower than the calculated one. This can be explained by optical losses within the probes and means that there is a room for improvements, which can come, for instance, from using customized optics for the light transfer within the probes and more efficient light coupling into the detection fiber bundle. Nevertheless, the relative responsivity shows that the OCTRaman probe has a comparable fiber bundle performance, and hence, this probe can be used for the in vivo skin Raman signal detection.

For the further evaluation of the Raman probe performance, the MPE values should be taken into account. MPE calculations (according to ANSI Z136.1 standard, as most restrictive out of three values,MPE for a single pulse, average MPE for a pulse, MPE for the exposure time divided by the number of the pulses within this exposure time, and MPE for the train of pulses) for the skin exposure of $10 \mathrm{~s}$ to a laser pulse trail of wavelength of $532 \mathrm{~nm}$ at $20 \mathrm{~Hz}$ repetition rate and $7 \mathrm{~ns}$ pulse duration show that the skin irradiance should not exceed $53.2 \mathrm{~J} \mathrm{~m}^{-2}$. This translates into $42 \mu \mathrm{J}$ per pulse for the fiber bundle probe, assuming that the light delivery fiber has a diameter of $1 \mathrm{~mm}$. For the confocal setup, the MPE threshold corresponds to $167 \mu \mathrm{J}$ per pulse, while for the OCT-Raman probe with the full field of view of $\varnothing 9.4 \mathrm{~mm}$ it can be used at $3.6 \mathrm{~mJ}$ per pulse, as given in Table I, leading to much higher Raman signals detected within the MPE limits.

Taking into account both the light detection efficiency and the limitation introduced by the MPE values, we can conclude that the OCT-Raman probe has superior performance to the fiber bundle and confocal probes for in vivo measurements and, hence, can be applied for the clinical use.

\section{COMBINED OCT-RAMAN PROBE IN VIVO TEST}

To demonstrate the applicability of the OCT-Raman probe, Raman spectra of human Caucasian skin were recorded in vivo at two different locations, volar and dorsal (palm and back) areas of a hand. The spectra were recorded for $100 \mathrm{~s}$, corresponding to calculated values of MPE of $30 \mathrm{~J} / \mathrm{m}^{2}$. This results in the laser pulse energy of $\sim 1.5 \mathrm{~mJ}$ for the illuminated area of the elliptic shape with axes of $9 \mathrm{~mm}$ and $7 \mathrm{~mm}$.

The recorded Raman spectra were processed to remove the luminescence background by means of an Improved Modified Multi-Polynomial Fitting (I-Mod-Poly) ${ }^{19}$ as shown in Fig. 3(a). Note that the baseline subtraction does not remove the shot noise caused by the fluorescence and, thus, does not improve the SNR. The SNR of the Raman spectrum, after background subtraction by the I-Mod-Poly method was calculated as a ratio of the root mean square amplitudes of the signal in the strongest line of the spectrum to the background and is equal to 18.7. The Raman spectrum SNR can be further improved with optimization of the Raman optical setup for the clinical trials. The resultant spectra look very similar to the in vivo skin Raman spectra reported previously, 4,20

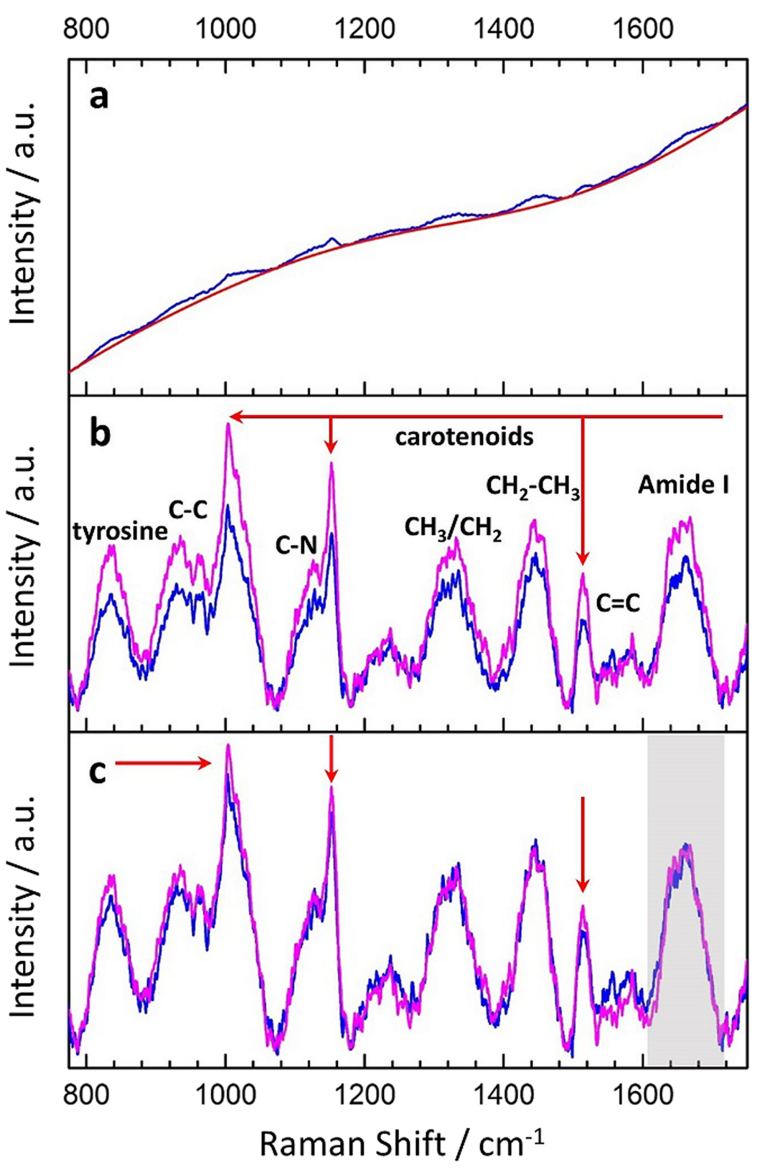

FIG. 3. (a) Raw in vivo spectrum (blue) and fitted luminescence baseline (red); (b) recorded Raman spectra of palm (magenta) and back of hand (blue) and Raman band assignment; (c) normalized on Amid I band (shaded area) area Raman spectra of palm (magenta) and back of hand (blue). Red arrows show a difference in carotenoid signals between two spectra. 
for example, with spectral lines and bands assigned as per Ref. 21.

From Fig. 3(b), it can be seen that the spectrum of the back of the hand has lower intensity compared to that of the palm. This is a result of the higher melanin concentration and, hence, higher skin absorption, in more tanned skin on the back of the hand. For the further comparison of the Raman spectra, they were normalized on the area of the Amid I band, Fig. 3(c). The normalized spectra show higher carotenoid concentration in the palm skin, as expected from the thicker stratum corneum layer, responsible for the carotenoid accumulation.

\section{CONCLUSION}

In this work, we report on the realization and evaluation of a combined OCT-Raman probe integrated into a spectral domain OCT measurement head. We show that the use of the full field of view of the scanning OCT lens allows for efficient detection of the Raman signal for in vivo skin measurements, at skin irradiance below MPE values with a good SNR. The performance of the system is compared to the most common Raman probe setups based on fiber bundles and confocal free space optics, respectively. We found that the combined probe has superior performance with respect to the reference systems. Also, the components for the Raman setup integrated in the OCT system do not affect the OCT performance. These features render the combined OCT-Raman probe applicable for future in vivo clinical trials. Currently, clinical trials on melanoma skin cancer detection and margin assessment are under way. Future work on the system includes the optimization of the clinical measurement procedure and the data analysis, the increase of the light collection efficiency and the SNR, and the development of a more compact and flexible probe.

\section{ACKNOWLEDGMENTS}

This work was supported by the German Federal Ministry of Education and Research (BMBF) within the VIP-project MeDiOO (Grant No. 03V0826).
${ }^{1}$ M. A. Calin, S. V. Parasca, R. Savastru, M. R. Calin, and S. Dontu, J. Cancer Res. Clin. Oncol. 139(7), 1083-1104 (2013).

${ }^{2}$ J. R. Maher, O. Chuchuen, M. H. Henderson, S. Kim, M. T. Rinehart, A. D. M. Kashuba, A. Wax, and D. F. Katz, Biomed. Opt. Express 6(6), 2022-2035 (2015).

${ }^{3}$ J. Qi, N. Sudheendran, C.-H. Liu, G. M. Santos, E. D. Young, A. J. Lazar, D. C. Lev, R. E. Pollock, K. V. Larin, and W.-C. Shih, Proc. SPIE 9318, 931807 (2015).

${ }^{4}$ C. A. Patil, H. Kirshnamoorthi, D. L. Ellis, T. G. van Leeuwen, and A. Mahadevan-Jansen, Lasers Surg. Med. 43(2), 143-151 (2011).

${ }^{5}$ V. P. Zakharov, I. A. Bratchenko, D. N. Artemyev, O. O. Myakinin, D. V. Kornilin, S. V. Kozlov, and A. A. Moryatov, J. Biomed. Opt. 20(2), 025003 (2015).

${ }^{6}$ P. C. Ashok, B. B. Praveen, N. Bellini, A. Riches, K. Dholakia, and C. S. Herrington, Biomed. Opt. Express 4(10), 2179-2186 (2013).

${ }^{7}$ J. Schleusener, C. Reble, M. C. Meinke, and J. Helfmann, Photonics Lasers Med. 4(2), 151-167 (2015).

${ }^{8}$ M. G. Shim, B. C. Wilson, E. Marple, and M. Wach, Appl. Spectrosc. 53(6), 619-627 (1999).

${ }^{9}$ T. Katagiri, Y. S. Yamamoto, Y. Ozaki, Y. Matsuura, and H. Sato, Appl. Spectrosc. 63(1), 103-107 (2009).

${ }^{10}$ S. Koljenovic, T. C. B. Schut, R. Wolthuis, B. de Jong, L. Santos, P. J. Caspers, J. M. Kros, and G. J. Puppels, J. Biomed. Opt. 10(3), 031116 (2005).

${ }^{11}$ N. D. Magee, J. S. Villaumie, E. T. Marple, M. Ennis, J. S. Elborn, and J. J. McGarvey, J. Phys. Chem. B 113(23), 8137-8141 (2009).

${ }^{12}$ M. G. Shim and B. C. Wilson, J. Raman Spectrosc. 28 (2-3), 131-142 (1997).

${ }^{13}$ J. Mo, W. Zheng, and Z. Huang, Biomed. Opt. Express 1(1), 17-30 (2010).

${ }^{14}$ J. Zhao, H. Lui, D. I. McLean, and H. Zeng, Skin Res. Technol. 14(4), 484-492 (2008).

${ }^{15}$ A. Mahadevan-Jansen, M. F. Mitchell, N. Ramanujam, U. Utzinger, and R. Richards-Kortum, Photochem. Photobiol. 68(3), 427-431 (1998).

${ }^{16}$ O. Stevens, I. E. I. Petterson, J. C. C. Day, and N. Stone, Chem. Soc. Rev. 45(7), 1919-1934 (2016).

${ }^{17}$ I. Pence and A. Mahadevan-Jansen, "Clinical instrumentation and applications of Raman spectroscopy," Chem. Soc. Rev. 45(7), 1958-1979 (2016).

${ }^{18}$ A. Varkentin, M. Mazurenka, E. Blumenröther, M. Meinhardt-Wollweber, M. Rahlves, S. M. C. Broekaert, S. Schäd-Trcka, S. Emmert, U. Morgner, and B. Roth, J. Biophotonics 10, 854-861 (2017).

${ }^{19}$ J. Zhao, H. Lui, D. I. McLean, and H. Zeng, Appl. Spectrosc. 61(11), 12251232 (2007).

${ }^{20}$ M. Gniadecka, P. A. Philipsen, S. Sigurdsson, S. Wessel, O. F. Nielsen, D. H. Christensen, J. Hercogova, K. Rossen, H. K. Thomsen, R. Gniadecki, L. K. Hansen, and H. C. Wulf, J. Invest. Dermatol. 122(2), 443-449 (2004).

${ }^{21}$ S. Tfaili, C. Gobinet, G. Josse, J.-F. Angiboust, M. Manfait, and O. Piot, Analyst 137(16), 3673-3682 (2012). 